\title{
ALMOST CONTINUITY OF THE CESȦRO-VIETORIS FUNCTION
}

\author{
JACK B. BROWN
}

ABSTRACT. Consider the following function due to Cesàro: $\phi(0)=0$, and if $0<x \leq 1$,

$$
\phi(x)=\lim \sup \left(a_{1}+a_{2}+\cdots+a_{n}\right) / n,
$$

where the $a_{i}$ are given by the unique nonterminating binary expansion of $x=\left(0 . a_{1} a_{2} \cdots\right)$. Vietoris proved in 1921 that $\phi$ is connected (as a subset of $[0,1] \times R)$. The purpose of this note is to alter Vietoris's argument in order to prove that $\phi$ is actually almost continuous in the sense of Stallings, thus answering a question raised recently by B. D. Smith.

B. D. Smith [7] recently proved that a real function $f:[0,1] \rightarrow R$ is continuous if and only if it satisfies the three conditions (i) $f$ is almost continuous in the sense of Stallings [8] (for any open set $N \subset[0,1] \times R$ containing $f, N$ contains a continuous function $g:[0,1] \rightarrow R$ ), (ii) $f$ is almost continuous in the sense of Husain [3] (for every $x \in[0,1]$ and open set $V \subset R$ containing $f(x), \mathrm{Cl}\left(f^{-1}(V)\right)$ is a neighborhood of $\left.x\right)$, and (iii) $f$ is not of Cesàro type (for every open $U \subset[0,1]$ and open $V \subset R$ there exists $y \in V$ such that $\left.U \not \subset \mathrm{Cl}\left(f^{-1}(y)\right)\right)$. In investigating the possible redundancy of (i), (ii), and (iii), Smith leaves open only the question of whether (i) and (ii) imply (iii). He poses as a very likely counterexample to this implication the function $\phi$ of Cesàro. It satisfies (ii) but not (iii). Vietoris [9] showed that $\phi$ is connected, and Smith points out that Stallings [8] raised the question of whether connected functions from $[0,1]$ into $R$ necessarily satisfy (i). If such were the case, then $\phi$ would necessarily satisfy (i). However, this question of Stallings has been answered in the negative. The first counterexample, due to Jones and Thomas [4], happens to satisfy (iii), but later examples [1], [2], [6], satisfy (ii) but not (iii), are connected, but do not satisfy (i). This casts some doubt as to whether $\phi$ might satisfy (i).

Received by the editors February 1, 1974.

AMS (MOS) subject classifications (1970). Primary 26A15, 54C10, 54C 30. function.

Key words and phrases. Almost continuous, connected graph, Cesàro-Vietoris 
It might be said here that it is possible to use standard transfinite techniques to construct a function $f$ which intersects every closed subset $C$ of $[0,1] \times R$ which has a nondegenerate connected projection onto $[0,1]$. Then, $f$ would satisfy (ii) but not (iii), and it would follow from the theorem about "minimal blocking sets" of [5] that $f$ would satisfy (i). However, it seems desirable to just determine whether the simpler and oft-used function $\phi$ satisfies (i).

Theorem. The Cesaro function $\phi$ is almost continuous in the sense of Stallings.

Proof. Vietoris's proof that $\phi$ has a connected graph [9, pp. 202-204] will be referred to extensively. Suppose $\phi$ is not almost continuous in the sense of Stallings, and let $O$ be an open subset of $[0,1] \times R$ containing $\phi$ but containing no continuous function $g:[0,1] \rightarrow R$. Let $N$ be a neighborhood of $(0,0)$ which lies in $O$, assume without loss of generality that $N \cap Y=O \cap Y$ (Y is the $y$-axis), and define $G=\{(x, y) \mid(x, y) \in N$ or else $0<x$ and there exists a continuous function $g:[0, x] \rightarrow R$ lying in $O$ such that $g(x)=y\}$. $G$ is obviously connected. It is also open, for suppose $(x, y)$ $\epsilon G$ and $g:[0, x] \rightarrow R$ is a continuous function lying in $O$ such that $g(x)=$ $y$. Consider a rectangle $s$ with center $(x, y)$, interior lying in $O$, and having a vertical left edge that intersects $g$ in a point $\left(x^{\prime}, y^{\prime}\right)$. Clearly the function $g^{\prime}=g \mid\left[0, x^{\prime}\right]$ can be extended continuously within $O$ to any point interior to $s$, so that the interior of $s$ lies in $G$. Let $H$ denote the boundary of $G$ and $J=[0,1] \times R-(G \cup H)$. Let $b=\sup \{x \mid(x, \phi(x)) \in G\}$. Clearly $b>0$. Suppose $(b, \phi(b))$ belongs to $G$ and let $g:[0, b] \rightarrow R$ be a continuous function lying in $O$ such that $g(b)=\phi(b)$. Then $b<1$, because by supposition there is no continuous $g:[0,1] \rightarrow R$ lying in $O$. Then a spherical neighborhood containing $(b, \phi(b))$ and lying in $O$ will contain a point $(c, \phi(c))$ with $b<c$, and the function $g$ can be continuously extended within that neighborhood to $(c, \phi(c))$. Thus, $(c, \phi(c))$ is a point of $G$ with abscissa greater than $b$, and this is a contradiction. So $(b, \phi(b))$ does not belong to $G$.

Now consider a spherical neighborhood $N^{\prime}$ of $(b, \phi(b))$ lying in $O$ and having radius $r$. Notice that every point in the left half of $N^{\prime}$ belongs to $J$, because otherwise the left half of $N^{\prime}$ would necessarily contain a point $\left(x^{\prime}, y^{\prime}\right)$ of $G$ and the continuous function $g^{\prime}$ associated with $\left(x^{\prime}, y^{\prime}\right)$ could be extended continuously within $O$ to $(b, \phi(b))$. Now, let $(a, \phi(a))$ be a point of $G$ such that $b-r<a<b$. Now consider a neighborhood $N^{\prime \prime}$ of 
$(a, \phi(a))$ of radius $r^{\prime}<\min (b-a, a-b+r)$ such that $N^{\prime \prime}$ lies in $G$. Then, the vertical strip $W$ of all points in $[0,1] \times R$ with abscissa in $\left[a, a+r^{\prime}\right]$ is such that every vertical line lying in $W$ contains a point of $G$ with a positive ordinate and a point of $J$ with a positive ordinate and therefore also a point of $H$ with a positive ordinate. This is the point to which Vietoris arrives in his argument at the top of p. 204 of [9].

What follows is a paraphrasing of Vietoris's argument, altered slightly to serve the special purposes of this proof. Let $H_{0}=H \cap W$. Let $W_{1}, W_{2}, \ldots$ be a sequence of open sets containing $H_{0}\left(W_{n}\right.$ being the union of the $\left(1 / 2^{n}\right)$-neighborhoods of points of $\left.H_{0}\right)$. A sequence $n(1), n(2), \ldots$ of positive integers and a dyadic decimal $0 . a_{1} a_{2} \ldots$ will be defined simultaneously, with $M_{i}$ denoting $\left(a_{1}+a_{2}+\cdots+a_{i}\right) / i$ as the process is carried out. First pick a dyadic rational $\xi_{0}=0 . a_{1} \cdots a_{j}$ between $a$ and $a+r^{\prime}$ and then any point $c_{1}=\left(\xi_{0}, \eta_{0}\right)$ of $G \cap W_{1}$ with $\eta_{0}>0$. Consider a square shaped neighborhood $Q$ of $c_{1}$ which has radius $q<\eta / 2$ and lies interior to $G \cap W_{1} \cap W$. Now, proceed to define $a_{j+1}, a_{j+2}, \ldots$ etc., using consecutive 0 's up to a stage $a_{k}$ so that (1) regardless of how $0 . a_{1} \cdots a_{j} \cdots a_{k} \cdots$ is continued, it will differ from $\xi_{0}$ by less than $q$, (2) $M_{k}<q$ and (3) $1 / k$ $<q$. Then continue defining $a_{k+1}, a_{k+2}, \cdots$ etc., using consecutive 1's up to a stage $a_{n(1)}$ so that the point $b_{1}=\left(\xi_{1}, M_{n(1)}\right)$ is in $Q$ (where $\xi_{1}=$ $\left.0 . a_{1} a_{2} \cdots a_{n(1)}\right)$. This can be accomplished since $\left|M_{i+1}-M_{i}\right|<1 /(i+1)$ $<q$ for each $i \geq k$. Now repeat the process, starting with any point $c_{2}=$ $\left(\xi_{1}, \eta_{1}\right)$ of $G \cap W_{2}$ with $\eta_{1}>0$, and then defining $a_{n(1)+1}, a_{n(2)+2}, \ldots$ etc., first using consecutive 0 's and then using consecutive 1 's up to a stage $a_{n(2)}$ so that $\xi_{2}=0 . a_{1} a_{2} \cdots a_{n(2)}$ is between $\xi_{1}$ and $a+r^{\prime}$ and the point $b_{2}=\left(\xi_{2}, M_{n(2)}\right)$ is in $W_{2} \cap G$. Continue this process. Define $x_{\omega}=0 . a_{1} a_{2} \ldots$ and $y_{\omega}=\phi\left(x_{\omega}\right)$. Since consecutive 0 's and then consecutive 1 's were used in proceeding from $a_{n(r)}$ to $a_{n(r+1)}$ in the above process, it is true that for each $i$ between $n(r)$ and $n(r+1)$,

$$
M_{i} \leq \max \left\{M_{n(r)}, M_{n(r+1)}\right\}
$$

so that $\phi\left(x_{\omega}\right)=\lim \sup M_{i}=\lim \sup M_{n(i)}$. Therefore, the point $\left(x_{\omega}, y_{\omega}\right)$ is the limit of some subsequence of $b_{1}, b_{2}, \ldots$ Also, since $b_{n} \in W_{n}$, $\left(x_{\omega}, y_{\omega}\right) \in H_{0}$. Then since $\left(x_{\omega}, y_{\omega}\right)$ is a point of $\phi$, it has a neighborhood $N^{\prime \prime \prime}$ which lies in $O$, and $N^{\prime \prime \prime}$ contains one of the points $b_{i}$. Notice that $b_{i}$ lies to the left of $\left(x_{\omega}, y_{\omega}\right)$, so that the continuous function $g$ which lies in $O$, has domain an interval, and has left end $(0,0)$ and right end $b_{i}$ can 
be continuously extended within $O$ to $\left(x_{\omega}, y_{\omega}\right)$. So $\left(x_{\omega}, y_{\omega}\right)$ belongs to $G$ rather than $H$. This is a contradiction.

Remark. It is to a certain degree disappointing that $\phi$ satisfies (i) because it would be interesting to find a function which has a connected graph, and does not satisfy (i), but which could be described in terms of an equation (rather than by the topological techniques of [4] or the transfinite techniques of [1], [2], and [6]).

\section{REFERENCES}

1. J. B. Brown, Connectivity, semi-continuity, and the Darboux property, Duke Math. J. 36 (1969), 559-562. MR 39 \#7568.

2. J. L. Cornette, Connectivity functions and images on Peano continua, Fund. Math. 58 (1966), 183-192. MR 33 \#6600.

3. T. Husain, Almost continuous mappings, Prace Mat. 10 (1966), 1-7. MR $36 \# 3322$.

4. F. B. Jones and E. S. Thomas, Jr., Connected $G_{\delta^{-g r a p h s}}$, Duke Math. J. 33 (1966), 341-345. MR $33 \# 702$.

5. K. R. Kellum and B. D. Garrett, Almost continuous real functions, Proc. Amer. Math. Soc. 33 (1972), 181-185. MR 45 \# 2106.

6. J. H. Roberts, Zero-dimensional sets blocking connectivity functions, Fund. Math. 57 (1965), 173-179. MR 33 \#3270.

7. B. D. Smith, An alternate characterization of continuity, Proc. Amer. Math. Soc. 39 (1973), 318-320. MR 47 \# 4202.

8. J. Stallings, Fixed point theorems for connectivity maps, Fund. Math. 47 (1959), 249-263. MR 22 \#8485.

9. L. Vietoris, Stetige Mengen, Monatsh. Math. Phys. 31 (1921), 173-204.

DEPARTMENT OF MATHEMATICS, AUBURN UNIVERSITY, AUBURN, ALABAMA 36830 